\title{
miR-140-5p inhibits human glioma cell growth and invasion by targeting JAG1
}

\author{
HUAN-LI YANG ${ }^{1}$, YA-MEI GAO ${ }^{2}$ and JIN-AN ZHAO ${ }^{3}$ \\ ${ }^{1}$ Disinfection Supply Room; ${ }^{2}$ Nursing Department; ${ }^{3}$ Department of Neurosurgery, \\ Weinan Central Hospital, Weinan, Shaanxi 714000, P.R. China
}

Received April 16, 2016; Accepted March 16, 2017

DOI: $10.3892 / \mathrm{mmr} .2017 .6951$

\begin{abstract}
R-140-5p has been reported to be a tumor suppressor in several types of human cancer, however, little is known about its expression and function in human gliomas. The present study aimed to detect the expression of miR-140-5p in human glioma tissues and cell lines, and to investigate the effect of miR-140-5p on glioma cell growth, invasion and adhesion using in vitro gain-of-function and loss-of-function experiments. Furthermore, the hypothesis that Jagged1 (JAG1) may be a target gene of miR-140-5p was tested. Reverse transcription-quantitative polymerase chain reaction analysis revealed that miR-140-5p was significantly downregulated in human glioma tissues and cell lines compared with normal tissues, and that its expression was correlated with the grade of gliomas. Transfection of a miR-140-5p mimic into SW1783 glioma cells promoted cell growth, invasion and adhesion, as determined by MTT, Transwell and cell adhesion assays respectively. By contrast, transfection of a miR-140-5p inhibitor had the opposite effect. A dual-luciferase reporter assay confirmed that JAG1 was a target gene of miR-140-5p, and miR-140-5p inhibited JAG1 expression both at the mRNA and protein level. In addition, JAG1 overexpression reversed the effect of miR-140-5p on glioma cell growth, invasion and adhesion. In conclusion, the present study is the first to reveal that miR-140-5p acts as a tumor suppressor in human gliomas. JAG1 was demonstrated to be a novel target of miR-140-5p, and miR-140-5p exerted its inhibitory effect on human glioma growth and invasion, partly by suppressing JAG1. The present study may provide useful information toward novel targets for the treatment of gliomas.
\end{abstract}

Correspondence to: Dr Jin-An Zhao, Department of Neurosurgery, Weinan Central Hospital, West Victory Street, Weinan, Shaanxi 714000, P.R. China

E-mail: zhaojinan_6671@sina.cn

Key words: glioma, miR-140-5p, Jagged 1, growth, invasion

\section{Introduction}

Gliomas account for approximately $30 \%$ of all brain and central nervous system tumors, and $80 \%$ of all malignant brain tumors (1). Although conventional treatments for gliomas are effective in controlling the disease, the median survival time of patients with gliomas is only 15 months (2). Exceptional invasiveness is one of the major reasons for treatment failure. Therefore, it is necessary to elucidate the molecular mechanisms for glioma invasion, and to find novel targets for the treatment of gliomas.

Recently, the association between microRNAs (miRNAs) and tumorigenesis has become a hot topic in the field of cancer research (3-5). miRNAs are small, non-coding RNAs which regulate a wide range of cellular processes, including cell survival, death, differentiation, and motility $(6,7)$. miRNAs act as tumor oncogenes or suppressors in various types of cancer (8-11), including gliomas (12-16). miR-140-5p has been reported to be a tumor suppressor in hepatocellular carcinoma (17), tongue squamous cell carcinoma (18), non-small cell lung cancer (19), and colorectal cancer (20). Recent research has demonstrated that the gene expression of hsa-miR-140-5p correlates with several selected magnetic resonance imaging features of patients with glioblastoma multiforme (21). However, little is known about the expression and the function of miR-140-5p in human gliomas.

The Notch signaling pathway participates in various aspects of tumor biology, and it has attracted increasing attention as a therapeutic target for cancer (22). Jagged1 (JAG1) is one of the canonical ligands for Notch receptors. It binds to Notch receptors and triggers Notch activation (23). Previous studies have demonstrated that JAG1 acts as an oncogene in brain tumors (24-26). JAG1 is overexpressed in glioblastoma blood vessels, and it promotes tumor growth as well as maintains cancer stem-like cells $(24,25,27)$.

In the present study, the expression of miR-140-5p was detected in human glioma tissues and cell lines and compared to that of normal tissue. In vitro gain-of-function and loss-of-function experiments were then performed to investigate the effect of miR-140-5p on glioma cell growth, invasion and adhesion. Furthermore, the hypothesis that JAG1 may be a target of miR-140-5p was tested. The present study may contribute to developing novel therapeutic strategies for gliomas. 


\section{Materials and methods}

Tissue samples. The study was approved by the Ethics Committee of Weinan Central Hospital (Weinan, China). A total of 48 glioma tissue samples and 48 adjacent normal tissue samples were collected from 48 patients with glioma who underwent surgery at Weinan Central Hospital between March 2012 and July 2015, including 21 patients who had low-grade (grades I-II) and 27 patients who had high-grade (grades III-IV) glioma. Among these patients, 23 were male and 25 were female, with the age range 30-69 years. All patients signed informed consent prior to enrollment in the study.

Cell culture. The human glioma cell lines (U-87, LN-18, U-118, U-138 and SW1783) and the human embryonic kidney HEK293 cell line were purchased from the American Type Culture Collection (Manassas, VA, USA). The normal human astrocytes were purchased from ScienCell Research Laboratories, Inc. (Carlsbad, CA, USA). The cells were cultured in Dulbecco's modified Eagle's medium (DMEM; Thermo Fisher Scientific, Inc., Waltham, MA, USA) supplemented with $10 \%$ fetal bovine serum (FBS; Thermo Fisher Scientific, Inc.) in a humidified atmosphere of $5 \% \mathrm{CO}_{2}$ at $37^{\circ} \mathrm{C}$.

Cell transfection. The miR-control (5'-UUGUACUACACA AAAGUACUG-3'), miR-140-5p mimic (sense 5'-CAGUGG UUUUACCCUAUGGUAG-3'; antisense 5'-ACCAUAGGG U AAAACCACUGUU-3'), miR-140-5p inhibitor (antisense 5'-CUACCAUAGGGUAAAA CCACUG-3'), control empty vector (cat. no. v855-20; Invitrogen; Thermo Fisher Scientific, Inc.), JAG1 overexpression plasmid (JAG1-pcDNA3.1), JAG1 small interfering (si) RNA (sense 5'-GAUCUAAAAGGGAAU AAAAGGTT-3'; antisense 5'-CCUUUUAUUCCCUUUUAG AUCTT-3') and scramble siRNA (sense 5'-UUCUCCGAA CGUGU CACGUTT-3'; antisense 5'-ACGUGACACGUUCGG AGAATT-3') were synthesized from Sangon Biotech Co., Ltd. (Shanghai, China). JAG1 overexpression plasmid was synthesized by ligating the cDNA of JAG1 into pcDNA3.1 vector. They were transfected into the cells using Lipofectamine 2000 (Thermo Fisher Scientific, Inc.), following the manufacturer's instructions. Briefly, $50 \mathrm{nM}$ RNA oligonucleotides or $100 \mathrm{ng}$ plasmids, and $10 \mu \mathrm{l}$ lipofectamine were diluted in $250 \mu \mathrm{l}$ Opti-MEM (Thermo Fisher Scientific, Inc.). Then they were mixed and incubated at room temperature for $20 \mathrm{~min}$ to form a complex. Cells $\left(1 \times 10^{5}\right)$ were incubated with the complex for $6 \mathrm{~h}$ and then the cells were maintained in fresh medium for at least $24 \mathrm{~h}$ prior to analyses.

Luciferase reporter assay. TargetScan 7.1 software (http://www.targetscan.org/vert_71/) was used to predict the targets of miR-140-5p, and JAG1 was found to be a target gene. The wild type and mutant 3'-untranslated region (UTR) of JAG1 were cloned from JAG1 cDNA (accession number: NM_000214.2) and inserted into the pmirGLO luciferase reporter vector (Promega Corporation, Madison, WI, USA) in the $\mathrm{XhoI} / \mathrm{XbaI}$ sites. Primers used in this study were: wild type, forward (F) 5'-CCGCTCGAGAACCAGCAACGATCA CAA-3' and reverse (R) 5'-TCTAGAGCAAAGCCGGTA GAACTACG-3'; mutant, F 5'-CCGCTCGAGCATAATACT
GTTACTACTGTAGATTTGA-3' and R 5'- TCTAGAGC AAAGCCGGTAGAACTACG-3'. The conditions for PCR amplification were: $95^{\circ} \mathrm{C}$ for $5 \mathrm{~min}$, followed by 32 cycles of $95^{\circ} \mathrm{C}$ for $20 \mathrm{sec}, 56^{\circ} \mathrm{C}$ for $30 \mathrm{sec}$, and $72^{\circ} \mathrm{C}$ for $70 \mathrm{sec}$. For the luciferase assay, the HEK 293 cells were transfected with the JAG1 3'UTR-pmirGLO plasmid and miR-140-5p mimic or miR-control, using Lipofectamine 2000. The pRL-TK Renilla luciferase reporter vector (Promega Corporation) was used as an internal control for normalization. Luciferase activity in the cells was determined using a Luciferase Reporter Assay system (Promega Corporation).

3-(4,5-dimethylthiazol-2-yl)-2,5-diphenyltetrazolium bromide (MTT) assay. MTT assay was used to determine cell growth by measuring the numbers of live cells over $96 \mathrm{~h}$. Cells were plated at a density of $1 \times 10^{4}$ cells/well, and the cells were allowed to grow for 24, 48, 72 and $96 \mathrm{~h}$ in 96-well plates, and then incubated with MTT (Sigma-Aldrich; Merck KGaA, Darmstadt, Germany) at $37^{\circ} \mathrm{C}$, according to the manufacturer's instructions. Following $4 \mathrm{~h}$, dimethyl sulfoxide was added to solubilize the formazan crystals. Absorbance at $570 \mathrm{~nm}$ was measured using a Multiskan Ascent plate reader (Thermo Fisher Scientific, Inc.). Results were calculated relative to optical density of blank cells at $570 \mathrm{~nm}$ at $24 \mathrm{~h}$.

Transwell invasion assay. Transwell invasion assays were used to measure cell invasion ability. The Transwell inserts (Corning, Inc., Corning, NY, USA) were precoated with Matrigel (BD Biosciences, Franklin Lakes, NJ, USA) at $37^{\circ} \mathrm{C}$ for $30 \mathrm{~min}$. The resuspended cells $(2 \mathrm{ml})$ at the final concentration of $5 \times 10^{4}$ cells $/ \mathrm{ml}$ in serum-free medium were added to the upper chambers, and cell culture medium containing 10\% FBS was added to the lower chambers. Following incubation at $37^{\circ} \mathrm{C}$ for $12 \mathrm{~h}$, a cotton swab was used to gently remove the non-invasive cells which remained on the upper surface of the membrane. The invasive cells which migrated on the lower surface of the membrane were fixed with $95 \%$ ethanol for $30 \mathrm{~min}$, and then stained with hematoxylin (Beyotime Institute of Biotechnology, Shanghai, China) for $10 \mathrm{~min}$ at room temperature. The number of invaded cells was counted in 10 randomly selected fields using an inverted light microscope (37XC; Shanghai Optical Instrument Co., Ltd., Shanghai, China). Results are presented as the mean number of cells per field.

Cell adhesion assay. Cell adhesion was examined using 96-well plates coated with fibronectin (Sigma-Aldrich; Merck KGaA). The plates were blocked with $1 \%$ bovine serum albumin (BSA; Sigma-Aldrich; Merck KGaA) at room temperature for $2 \mathrm{~h}$. The cells were suspended to the density of $3 \times 10^{5}$ cells $/ \mathrm{ml}$, and $200 \mu \mathrm{l}$ cell suspension were cultured in serum-free medium at $37^{\circ} \mathrm{C}$ for $2 \mathrm{~h}$. After washing with PBS, the adhesive cells were fixed with $4 \%$ paraformaldehyde (Sangon Biotech Co., Ltd.) for $15 \mathrm{~min}$ and then stained with $0.1 \%$ crystal violet (Sangon Biotech Co., Ltd.) for 20 min. 12\% SDS was added to dissolve the crystals and the absorbance at $570 \mathrm{~nm}$ was measured using a microplate reader. Results were calculated relative to optical density at $570 \mathrm{~nm}$ of blank cells.

Western blot analysis. The proteins were extracted from the cells using Western and Immunoprecipitation Cell Lysis 

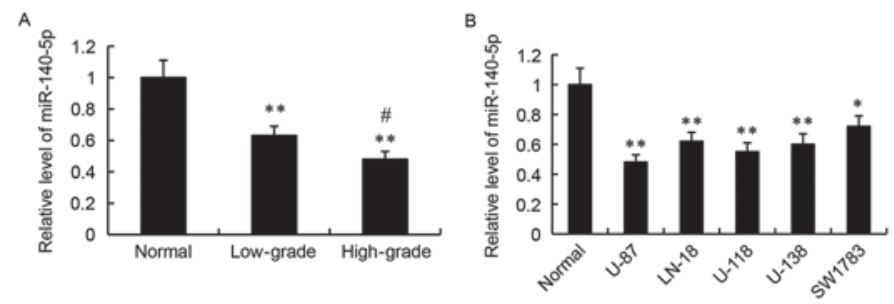

Figure 1. Expression of miR-140-5p in human glioma tissues and cell lines. $n=3$. (A) Relative levels of miR-140-5p in human glioma tissues and adjacent normal tissues. ${ }^{* *} \mathrm{P}<0.01$ compared with normal; ${ }^{*} \mathrm{P}<0.05$ compared with low-grade gliomas. (B) Relative levels of miR-140-5p in human glioma cell lines and in a human normal astrocyte cell line. ${ }^{*} \mathrm{P}<0.05$ and ${ }^{* *} \mathrm{P}<0.01$ compared with normal.

A
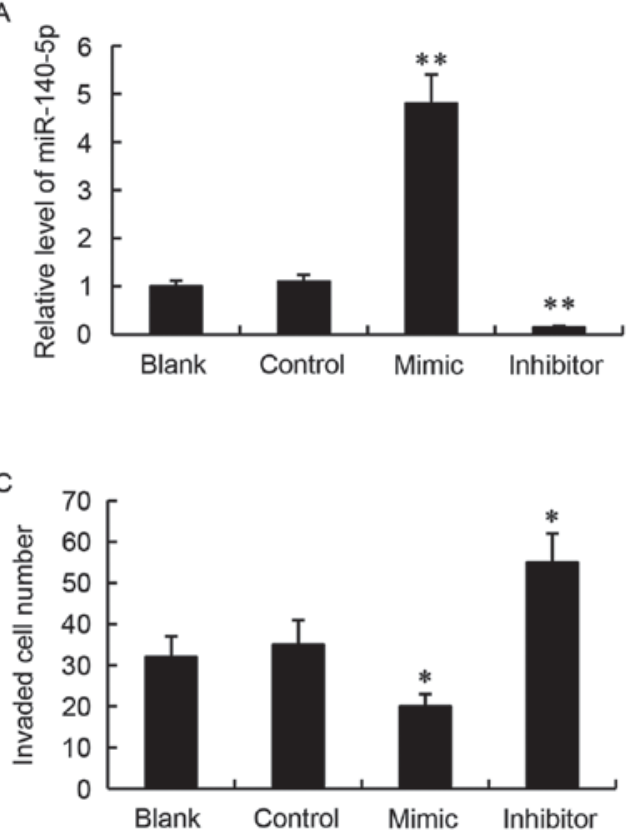
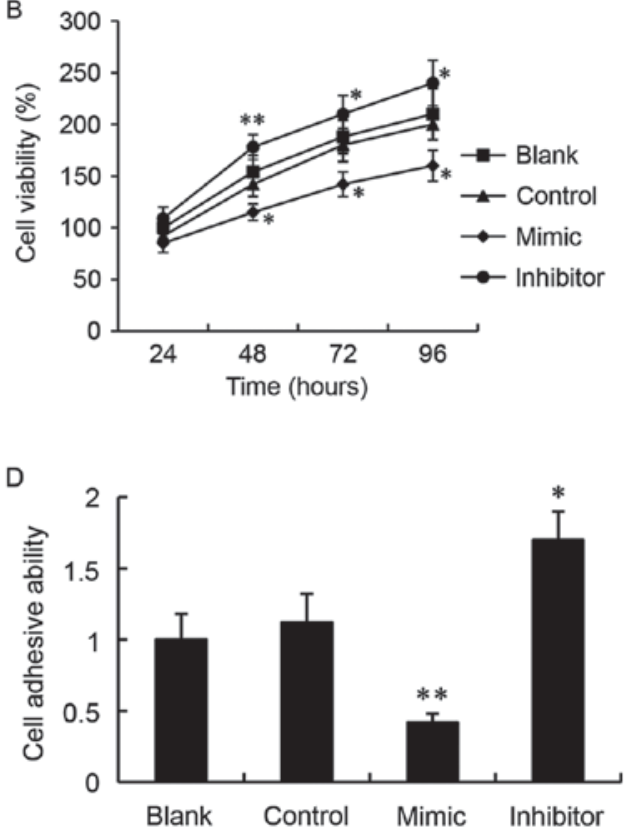

Figure 2. Effect of miR-140-5p on glioma cell growth, invasion and adhesion. SW1783 glioma cells were transfected with miR-control, miR-140-5p mimic and miR-140-5p inhibitor. Untransfected cells were used as the blank control. n=3. (A) Relative expression levels of miR-140-5p, (B) cell viability, (C) cell invasion and (D) cell adhesion were measured in the four experimental groups. ${ }^{*} \mathrm{P}<0.05$ and ${ }^{* * *} \mathrm{P}<0.01$ compared with control.

buffer (Sangon Biotech Co., Ltd.). Briefly, $5 \times 10^{5}$ cells were incubated with $50 \mu \mathrm{l}$ cell lysis buffer at $4^{\circ} \mathrm{C}$ for $20 \mathrm{~min}$. and protein concentrations were determined using a bicinchoninic acid protein assay kit (Sangon Biotech Co., Ltd.). Equal amounts of total protein $(20 \mu \mathrm{g})$ were separated on $8 \%$ SDS polyacrylamide gel electrophoresis, and then transferred to nitrocellulose membranes (Merck KGaA). Following blocking in tris-buffered saline containing $3 \% \mathrm{BSA}$ at $4^{\circ} \mathrm{C}$ overnight, the membranes were incubated with rabbit polyclonal antibody to JAG1 (1:500 dilution; cat no. ab7771; Abcam, Cambridge, MA, USA) and rabbit polyclonal antibody to $\beta$-actin (1:1,000 dilution; cat no. ab8227; Abcam) at $4^{\circ} \mathrm{C}$ overnight. Subsequently, the membranes were incubated with goat anti-rabbit horseradish peroxidase-conjugated secondary antibody $(1: 2,000$ dilution; cat no. ab205718; Abcam) at room temperature for $2 \mathrm{~h}$. The signals were detected using a chemiluminescence detection kit (Pierce; Thermo Fisher Scientific, Inc.), and band intensities were quantified using ImageJ version 1.46 (National Institutes of Health, Bethesda, MD, USA) (28).

Reverse transcription-quantitative polymerase chain reaction (RT-qPCR). Total RNA was isolated from the cells using
TRIzol reagent (Thermo Fisher Scientific, Inc.), and miRNAs were isolated from tissues and cells using the miRNeasy Mini kit (Qiagen GmbH,Hilden, Germany), as per the manufacturer's instructions. Total RNA ( $1 \mu \mathrm{g})$ was converted into cDNA using a RevertAid First Strand cDNA Synthesis kit (Thermo Fisher Scientific, Inc.), as per the manufacturer's instructions. Dilute the cDNA samples at 1:5 in RNAse free water. Subsequently, the qPCR reactions were carried out using a SYBR-Green PCR Master Mix (Applied Biosystems; Thermo Fisher Scientific, Inc.) in a 7900 Real-Time PCR system (Applied Biosystems; Thermo Fisher Scientific, Inc.). The primers used in this study were: miR-140-5p, F 5'-CAGTGGTTTTACCCTATGGTAG-3' and R 5'-TGGTGTCGTGGAGTCG-3'; U6, F 5'-CTCGCT TCGGCAGCACA-3' and R 5'AACGCTTCACGAATTTGC GT-3'; JAG1, F 5'-GACTCATCAGCCGTGTCTCA-3' and R 5'-TGGGGAACACTCACACTCAA-3'; $\beta$-actin, F 5'-GGA CTTCGAGCAAGAGATGG-3' and R 5'-AGCACTGTGTTG GCGTACAG-3'. The conditions for PCR amplification were: $95^{\circ} \mathrm{C}$ for $5 \mathrm{~min}$, followed by 40 cycles of $95^{\circ} \mathrm{C}$ for $20 \mathrm{sec}$, $60^{\circ} \mathrm{C}$ for $30 \mathrm{sec}$, and $72^{\circ} \mathrm{C}$ for $30 \mathrm{sec}$. The U6 spliceosomal RNA gene or the $\beta$-actin gene were used as internal controls for miRNA and mRNA expression respectively. The relative 
mRNA expression levels were calculated in accordance with the $2^{-\Delta \Delta \mathrm{Cq}}$ method (29).

Statistical analysis. Data are presented as the mean \pm standard deviation of three independent experiments. Statistical analysis was performed using SPSS 19.0 statistical software (IBM SPSS, Armonk, NY, USA). Student's t-test or one-way analysis of variance followed by least significant difference test were used to analyze the statistical difference between groups. $\mathrm{P}<0.05$ was considered to indicate a statistically significant difference.

\section{Results}

Expression of miR-140-5p in human glioma tissues and cell lines. RT-qPCR was used to detect the relative expression levels of miR-140-5p in human glioma tissues and cell lines. Compared with adjacent normal tissues, the relative expression levels of miR-140-5p were significantly decreased in the low-grade and high-grade human glioma tissues $(\mathrm{P}<0.01$; Fig. 1A). Furthermore, miR-140-5p expression was significantly lower in the high-grade gliomas compared with the low-grade gliomas ( $\mathrm{P}<0.05$; Fig. 1A). Next, miR-140-5p expression was examined in normal human astrocytes and in five glioma cell lines, U-87, LN-18, U-118, U-138 and SW1783. As demonstrated in Fig. 1B, the relative expression levels of miR-140-5p was significantly lower in the glioma cell lines compared with the normal human astrocytes.

Effect of miR-140-5p on glioma cell growth, invasion and adhesion. A miR-140-5p mimic or a miR-140-5p inhibitor was transfected into the SW1783 glioma cells in vitro, in order to overexpress or suppress miR-140-5p expression respectively. As demonstrated by RT-qPCR analysis, transfection with a miR-control did not alter miR-140-5p expression compared with the untransfected blank group (Fig. 2A). However, the relative expression levels of miR-140-5p were significantly increased in the mimic group and significantly decreased in the inhibitor group, compared with the miR-control group ( $\mathrm{P}<0.01$; Fig. $2 \mathrm{~A})$.

The effect of miR-140-5p on glioma cell growth was determined by MTT assay. The results demonstrated that transfection with the miR-140-5p mimic significantly decreased the number of live cells over $96 \mathrm{~h}$, while transfection with the miR-140-5p inhibitor significantly increased the number of live cells, compared with the miR-control group (Fig. 2B).

Transwell invasion assay was used to examine the ability of the cells to invade through matrigel. The results demonstrated that, compared with the control, miR-140-5p mimic transfection resulted in decreased numbers of invaded cells, while miR-140-5p inhibitor transfection had the opposite effect $(\mathrm{P}<0.05$; Fig. 2C).

Finally, a cell adhesion assay revealed that, compared with the cells transfected with the miR-control, the cells transfected with the miR-140-5p mimic exhibited decreased cell adhesive ability $(\mathrm{P}<0.01$; Fig. 2D). By contrast, the cell adhesive ability was significantly increased in the cells transfected with the miR-140-5p inhibitor compared with the miR-control group $(\mathrm{P}<0.05$; Fig. 2D).

Effect of miR-140-5p on JAG1 expression in glioma cells. JAG1 was predicted to be a target gene of miR-140-5p by using

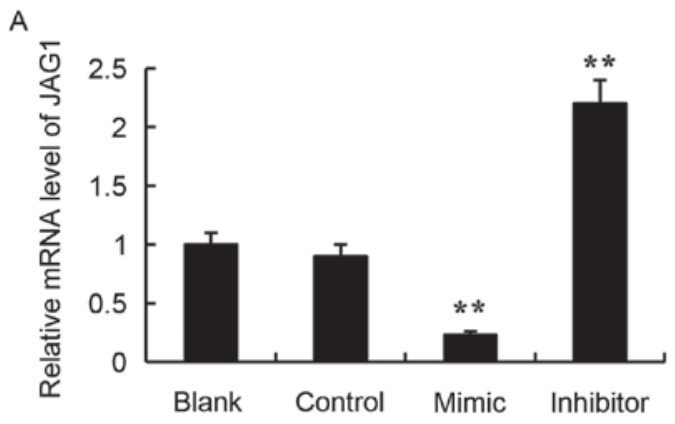

B
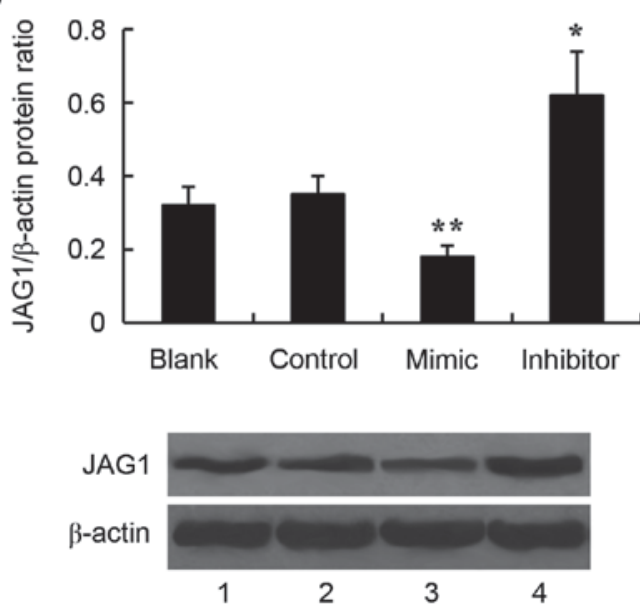

Figure 3. Effect of miR-140-5p on JAG1 expression in glioma cells. $n=3$. (A) Relative mRNA expression levels and (B) relative protein expression levels of miR-140-5p in SW1783 cells transfected with miR-control, miR-140-5p mimic and miR-140-5p inhibitor. Untransfected cells were used as the blank control. Lane 1, blank; lane 2, control; lane 3, mimic; lane 4, inhibitor. ${ }^{*} \mathrm{P}<0.05$ and ${ }^{* * *} \mathrm{P}<0.01$ compared with control. JAG1, Jagged 1.

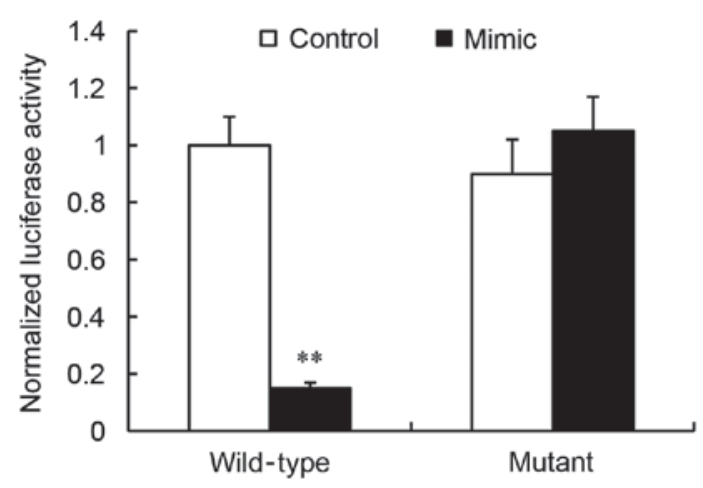

Figure 4. JAG1 is a direct target of miR-140-5p. The normalized luciferase activity was measured in HEK293 cells transfected with the wild-type or mutant JAG1 3'UTR reporter plasmid and the miR-140-5p mimic or miR-control. $\mathrm{n}=3$. ${ }^{* *} \mathrm{P}<0.01$ compared with control. JAG1, Jagged 1; 3'UTR, 3 ' untranslated region.

TargetScan 7.1 software (http://www.targetscan.org/vert_71/). To verify the effect of miR-140-5p on JAG1 expression, the miR-140-5p mimic or the miR-140-5p inhibitor was transfected into the SW1783 glioma cells, and expression of JAG1 was examined by RT-qPCR and western blotting. Transfection with the miR-140-5p mimic significantly inhibited JAG1 expression compared with control, both at the mRNA and 
A
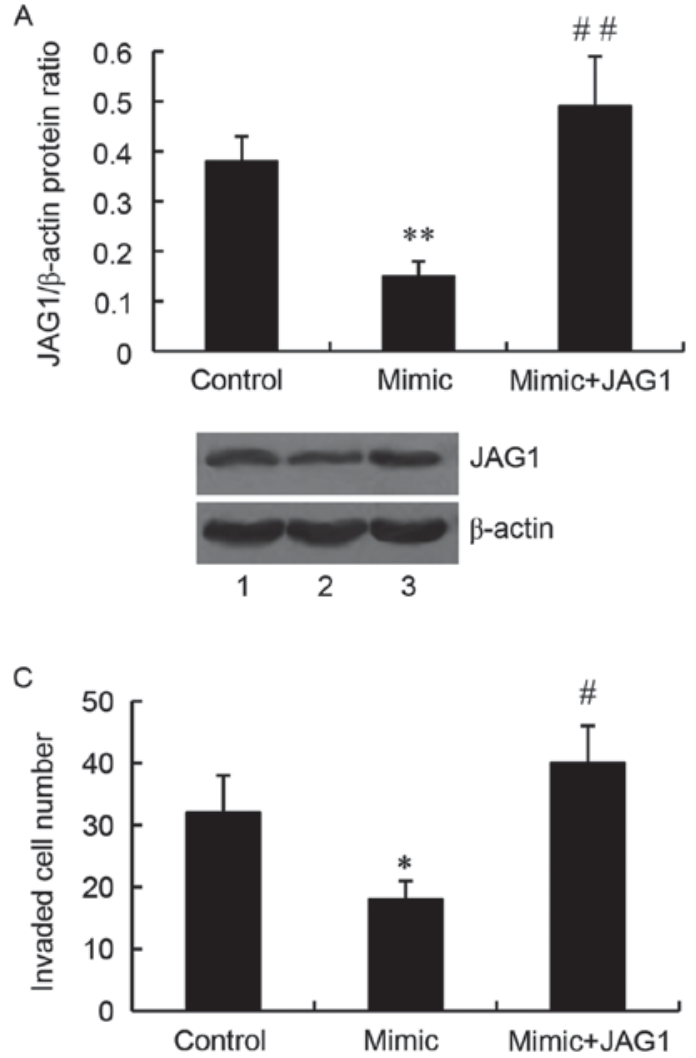
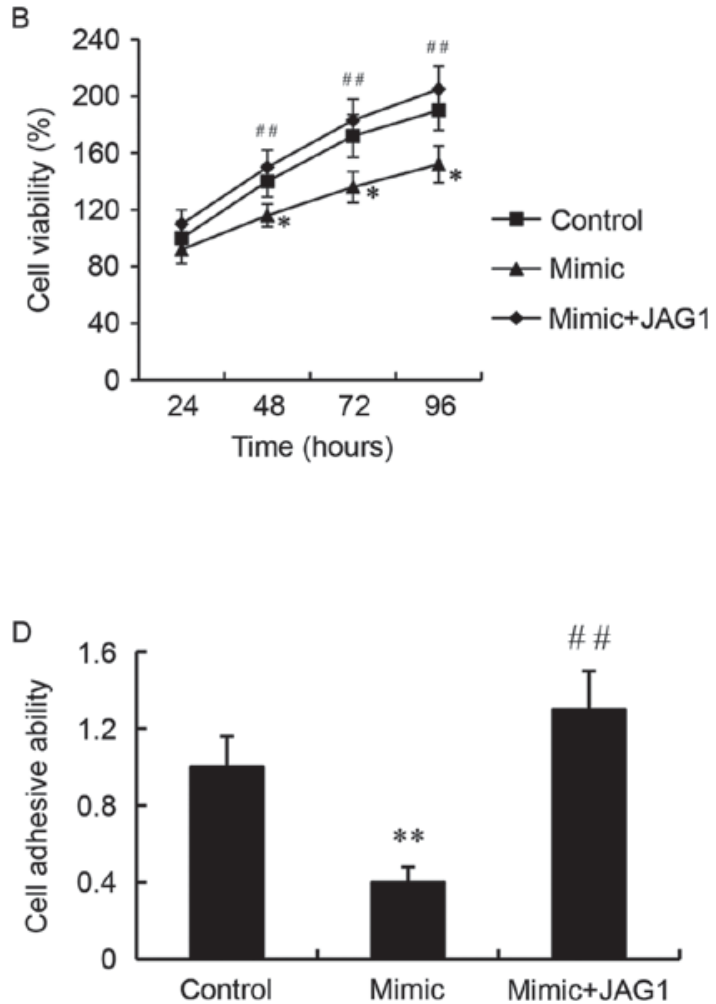

Figure 5. JAG1 overexpression reverses the effect of miR-140-5p mimic on glioma cell growth, invasion and adhesion. SW1783 glioma cells were transfected with the miR-140-5p mimic and/or the JAG1 overexpression plasmid. n=3. (A) Relative protein expression levels of JAG1 were determined by western blotting. Lane 1, control (cells transfected with miR-control and empty plasmid); lane 2, mimic; lane 3, mimic+JAG1. (B) Cell viability. (C) Cell invasion. (D) Cell adhesion. ${ }^{*} \mathrm{P}<0.05$ and ${ }^{* *} \mathrm{P}<0.01$ compared with control; ${ }^{\#} \mathrm{P}<0.05$ and ${ }^{\# \#} \mathrm{P}<0.01$ compared with the mimic. JAG1, Jagged 1.

A
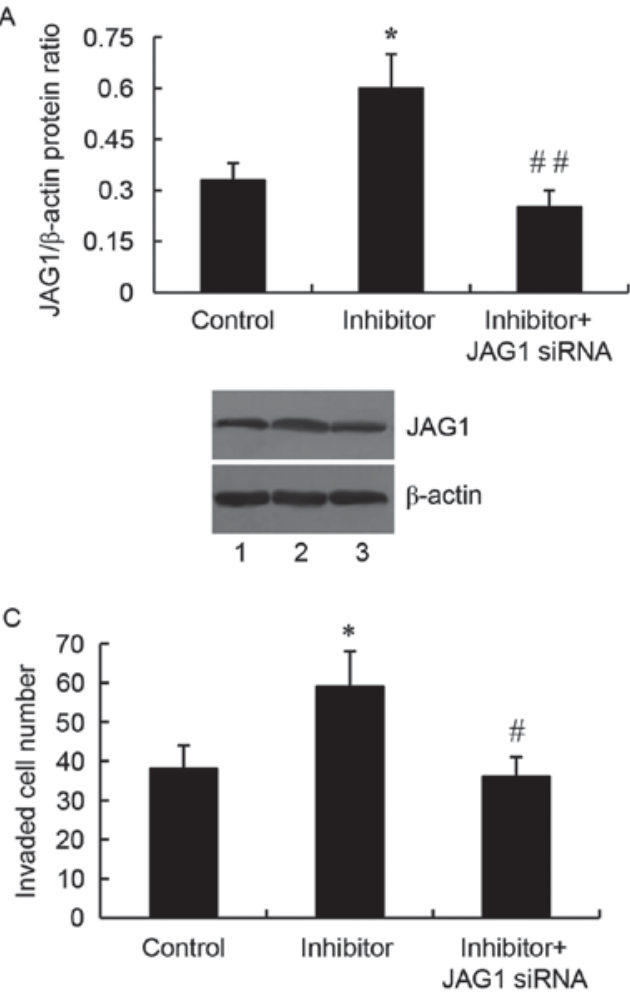

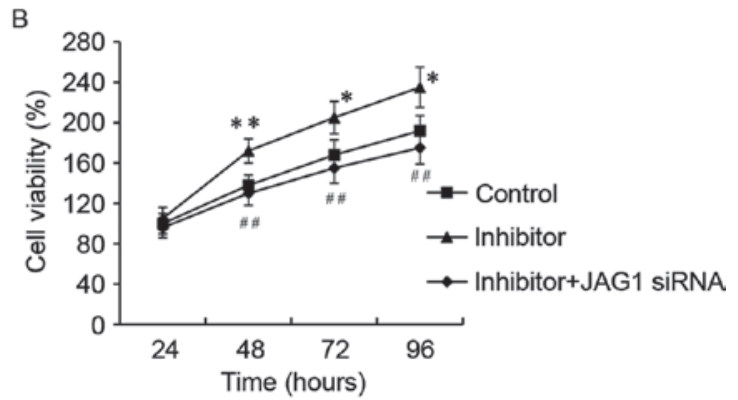

Figure 6. JAG1 silencing reverses the effect of miR-140-5p inhibitor on glioma cell growth, invasion and adhesion. SW1783 glioma cells were transfected with the miR-140-5p inhibitor and/or JAG1 siRNA. $n=3$. (A) Relative protein expression levels of JAG1 were determined by western blotting. Lane 1, control (cells transfected with miR-control and empty plasmid); lane 2, inhibitor; lane 3, inhibitor+JAG1 siRNA. (B) Cell viability. (C) Cell invasion. (D) Cell adhesion. ${ }^{*} \mathrm{P}<0.05$ and ${ }^{* *} \mathrm{P}<0.01$ compared with control; ${ }^{\#} \mathrm{P}<0.05$ and ${ }^{\# \#} \mathrm{P}<0.01$ compared with the inhibitor. JAG1, Jagged 1. 
protein level (Fig. 3). By contrast, transfection with the miR-140-5p inhibitor significantly increased JAG1 mRNA and protein expression levels, compared with control (Fig. 3).

JAG1 is a direct target of $m i R-140-5 p$. A dual-luciferase reporter assay was used in order to determine whether JAG1 was a direct target of miR-140-5p. The wild-type or mutant JAG1 3'UTR was cloned into the pmirGLO vector, and co-transfected into HEK293 cells with either the miR-140-5p mimic or the miR-control. The results revealed that, compared with control, transfection of the miR-140-5p mimic significantly repressed the luciferase activity of wild-type JAG1 3'UTR ( $\mathrm{P}<0.01$; Fig. 4). By contrast, transfection of the miR-140-5p mimic had no effect on the mutant JAG1 3'UTR luciferase activity compared with control (P>0.05; Fig. 4).

JAG1 mediates the effect of miR-140-5p on glioma cell growth, invasion and adhesion. JAG1 overexpression plasmid or JAG1 siRNA was used to overexpress or silence JAG1 respectively, and they were transfected into SW1783 cells together with the miR-140-5p mimic or inhibitor. As demonstrated by western blot analysis, the relative protein level of JAG1 was significantly higher in the cells transfected with the miR-140-5p mimic and the JAG1 overexpression plasmid, compared with the cells transfected with the mimic alone $(\mathrm{P}<0.01$; Fig. 5A). Importantly, JAG1 overexpression attenuated the inhibitory effect of miR-140-5p on glioma cell growth (Fig. 5B), invasion (Fig. 5C) and adhesion (Fig. 5D).

By contrast, cells transfected with the miR-140-5p inhibitor and the JAG1 siRNA exhibited a significantly downregulated JAG1 protein expression compared with cells transfected with the inhibitor only $(\mathrm{P}<0.01$; Fig. 6A). As expected, JAG1 silencing by siRNA reversed the miR-140-5p inhibitor-mediated increased cell growth (Fig. 6B), invasion (Fig. 6C) and adhesion (Fig. 6D) in SW1783 glioma cells.

\section{Discussion}

Abnormal expression of miR-140-5p is implicated in several types of cancer $(17,30)$. miR-140-5p has been reported to suppress the growth and metastasis of hepatocellular carcinoma, and its expression levels are correlated with various clinical characteristics of hepatocellular carcinoma, including vein invasion, multiple nodules, capsular formation, as well as overall and disease-free survival (30). miR-140-5p affects the proliferation of lung cancer cells by regulating mitogen-activated protein kinase 1 signaling (19). Upregulation of miR-140-5p suppresses migration and invasion of $\mathrm{FaDu}$ hypopharyngeal carcinoma cells by inhibiting the ADAM metallopeptidase domain 10-mediated Notch1 signaling pathway, suggesting that miR-140-5p may have potential therapeutic applications in hypopharyngeal squamous cell carcinoma (31). Consistent with previous studies, the present study demonstrated that miR-140-5p was significantly downregulated in human glioma tissues and cell lines compared with normal tissues, indicating that miR-140-5p may be a tumor suppressor in human gliomas. In addition, miR-140-5p expression was significantly correlated with the grade of gliomas, suggesting that miR-140-5p may be important in glioma progression. Subsequently, in vitro gain-of-function and loss-of-function experiments were performed to investigate the effect of miR-140-5p on glioma cell growth, invasion and adhesion. The present results revealed that miR-140-5p suppressed cell growth, invasion and adhesion in SW1783 glioma cells, suggesting that miR-140-5p may have an inhibitory effect on glioma growth and metastasis. Consistent with the role of miR-140-5p in other types of cancer, this study demonstrated for the first time that miR-140-5p acts as a tumor suppressor in human gliomas.

JAG1 mediates multiple signaling pathways, and is important in both physiological and pathological conditions (32-37). It has been demonstrated that JAG1 is overexpressed in various types of cancer, and it is involved in several aspects of tumor biology, including cell growth, apoptosis, cancer stem cell maintenance, epithelial-mesenchymal transition and metastasis (38). Fiaschetti et al (39) revealed that JAG1 is overexpressed in the majority of medulloblastoma (MB), and JAG1 is crucial in maintaining Notch-related pro-survival functions in MB cells (39). In the present study, JAG1 expression was demonstrated to be regulated by miR-140-5p in glioma cells. JAG1 was predicted to be a target gene of miR-140-5p by using TargetScan 7.1 software (http://www.targetscan.org/vert_71/). Using a dual-luciferase reporter assay, the present study confirmed that miR-140-5p inhibited JAG1 expression by directly targeting the 3'UTR of JAG1. Furthermore, the effect of miR-140-5p on glioma cell proliferation, invasion and adhesion was reversed by JAG1 overexpression, suggesting that JAG1 mediated the effect of miR-140-5p on glioma cell growth and invasion.

In conclusion, the present study revealed that miR-140-5p was downregulated in human glioma tissues and cell lines compared with normal tissues. miR-140-5p exerted its inhibitory effect on glioma growth and invasion at least partly by suppressing JAG1 expression. The present study revealed a novel molecular mechanism underlying human glioma growth and invasion, and provided a potential novel target for the treatment of gliomas.

\section{References}

1. Dolecek TA, Propp JM, Stroup NE and Kruchko C: CBTRUS statistical report: Primary brain and central nervous system tumors diagnosed in the United States in 2005-2009. Neuro Oncol 14 (Suppl 5): v1-v49, 2012.

2. Louis DN, Ohgaki H, Wiestler OD, Cavenee WK, Burger PC, Jouvet A, Scheithauer BW and Kleihues P: The 2007 WHO classification of tumours of the central nervous system. Acta Neuropathol 114: 97-109, 2007.

3. Chi Y and Zhou D: MicroRNAs in colorectal carcinoma-from pathogenesis to therapy. J Exp Clin Cancer Res 35: 43, 2016.

4. Li Y and Sarkar FH: MicroRNA targeted therapeutic approach for pancreatic cancer. Int J Biol Sci 12: 326-337, 2016.

5. Kuninty PR, Schnittert J, Storm G and Prakash J: MicroRNA targeting to modulate tumor microenvironment. Front Oncol 6: 3, 2016.

6. Sun W, Julie Li YS, Huang HD, Shyy JY and Chien S: microRNA: A master regulator of cellular processes for bioengineering systems. Annu Rev Biomed Eng 12: 1-27, 2010.

7. Ambros V: The functions of animal microRNAs. Nature 431: 350-355, 2004.

8. Lu J, Getz G, Miska EA, Alvarez-Saavedra E, Lamb J, Peck D, Sweet-Cordero A, Ebert BL, Mak RH, Ferrando AA, et al: MicroRNA expression profiles classify human cancers. Nature 435: 834-838, 2005. 
9. Volinia S, Calin GA, Liu CG, Ambs S, Cimmino A, Petrocca F, Visone R, Iorio M, Roldo C, Ferracin M, et al: A microRNA expression signature of human solid tumors defines cancer gene targets. Proc Natl Acad Sci USA 103: 2257-2261, 2006

10. Hurst DR, Edmonds MD and Welch DR: Metastamir: The field of metastasis-regulatory microRNA is spreading. Cancer Res 69: 7495-7498, 2009.

11. Iorio MV and Croce CM: MicroRNA dysregulation in cancer: Diagnostics, monitoring and therapeutics. A comprehensive review. EMBO Mol Med 4: 143-159, 2012.

12. Manrique-Guzmán S: Biomarkers in high-grade gliomas: A systematic review. Gac Med Mex 152: 87-93, 2016 (In Spanish).

13. Silber J, James CD and Hodgson JG: microRNAs in gliomas: Small regulators of a big problem. Neuromolecular Med 11: 208-222, 2009.

14. Wong JW: MicroRNA-induced silencing of glioma progression. J Neurosci 30: 3868-3869, 2010.

15. Yang HW, Xing H and Johnson MD: A major role for microRNAs in glioblastoma cancer stem-like cells. Arch Pharm Res 38 : 423-434, 2015.

16. Costa PM, Cardoso AL, Mano M and de Lima MC: MicroRNAs in glioblastoma: Role in pathogenesis and opportunities for targeted therapies. CNS Neurol Disord Drug Targets 14: 222-238, 2015.

17. Yang H, Fang F, Chang R and Yang L: MicroRNA-140-5p suppresses tumor growth and metastasis by targeting transforming growth factor $\beta$ receptor 1 and fibroblast growth factor 9 in hepatocellular carcinoma. Hepatology 58: 205-217, 2013.

18. Kai Y, Peng W, Ling W, Jiebing $H$ and Zhuan B: Reciprocal effects between microRNA-140-5p and ADAM10 suppress migration and invasion of human tongue cancer cells. Biochem Biophys Res Commun 448: 308-314, 2014.

19. Li W and He F: Monocyte to macrophage differentiation-associated (MMD) targeted by miR-140-5p regulates tumor growth in non-small cell lung cancer. Biochem Biophys Res Commun 450: 844-850, 2014

20. Zhai H, Fesler A, Ba Y, Wu S and Ju J: Inhibition of colorectal cancer stem cell survival and invasive potential by hsa-miR-140-5p mediated suppression of Smad2 and autophagy. Oncotarget 6: 19735-19746, 2015.

21. Li WB, Chen HY, Zhang W, Yan W, Shi R, Li SW and Jiang T: Relationship between magnetic resonance imaging features and miRNA gene expression in patients with glioblastoma multiforme. Chin Med J (Engl) 126: 2881-2885, 2013.

22. Xiao YF, Yong X, Tang B, Qin Y, Zhang JW, Zhang D, Xie R and Yang SM: Notch and Wnt signaling pathway in cancer: Crucial role and potential therapeutic targets (Review). Int J Oncol 48 : 437-449, 2016

23. Shimizu K, Chiba S, Saito T, Kumano K and Hirai H: Physical interaction of Delta1, Jagged1, and Jagged2 with Notch1 and Notch3 receptors. Biochem Biophys Res Commun 276: 385-389, 2000

24. Zhu TS, Costello MA, Talsma CE, Flack CG, Crowley JG, Hamm LL, He X, Hervey-Jumper SL,Heth JA, Muraszko KM, et al: Endothelial cells create a stem cell niche in glioblastoma by providing NOTCH ligands that nurture self-renewal of cancer stem-like cells. Cancer Res 71: 6061-6072, 2011.

25. Jeon HM, Kim SH, Jin X, Park JB, Kim SH, Joshi K, Nakano I and Kim H: Crosstalk between glioma-initiating cells and endothelial cells drives tumor progression. Cancer Res 74: 4482-4492, 2014.
26. Purow BW, Haque RM, Noel MW, Su Q, Burdick MJ, Lee J, Sundaresan T, Pastorino S, Park JK, Mikolaenko I, et al: Expression of Notch-1 and its ligands, Delta-like-1 and Jagged-1, is critical for glioma cell survival and proliferation. Cancer Res 65: 2353-2363, 2005.

27. Jubb AM, Browning L, Campo L, Turley H, Steers G, Thurston G, Harris AL and Ansorge O: Expression of vascular Notch ligands Delta-like 4 and Jagged-1 in glioblastoma. Histopathology 60: 740-747, 2012.

28. Schneider CA, Rasband WS and Eliceiri KW: NIH Image to Image J: 25 years of image analysis. Nat Methods 9: 671-675, 2012.

29. Livak KJ and Schmittgen TD: Analysis of relative gene expression data using real-time quantitative PCR and the 2(-Delta Delta C(T)) Method. Methods 25: 402-408, 2001

30. Zhang W, Zou C, Pan L, Xu Y, Qi W, Ma G, Hou Y and Jiang P. MicroRNA-140-5p inhibits the progression of colorectal cancer by targeting VEGFA. Cell Physiol Biochem 37: 1123-1133, 2015.

31. Jing P, Sa N, Liu X, Liu X and Xu W: MicroR-140-5p suppresses tumor cell migration and invasion by targeting ADAM10-mediated Notch1 signaling pathway in hypopharyngeal squamous cell carcinoma. Exp Mol Pathol 100: 132-138, 2016.

32. Chen X, Stoeck A, Lee SJ, Shih IeM, Wang MM and Wang TL: Jagged 1 expression regulated by Notch 3 and Wnt/ $\beta$-catenin signaling pathways in ovarian cancer. Oncotarget 1: 210-218, 2010.

33. Yamamoto M, Taguchi Y, Ito-Kureha T, Semba K, Yamaguchi N and Inoue $\mathrm{J}$ : NF- $\mathrm{KB}$ non-cell-autonomously regulates cancer stem cell populations in the basal-like breast cancer subtype. Nat Commun 4: 2299, 2013.

34. Choi K, Ahn YH, Gibbons DL, Tran HT, Creighton CJ, Girard L, Minna JD, Qin FX and Kurie JM: Distinct biological roles for the notch ligands Jagged-1 and Jagged-2. J Biol Chem 284: 17766-17774, 2009.

35. Wang Z, Li Y, Banerjee S, Kong D, Ahmad A, Nogueira V, Hay N and Sarkar FH: Down-regulation of Notch-1 and Jagged-1 inhibits prostate cancer cell growth, migration and invasion, and induces apoptosis via inactivation of Akt, mTOR and NF-kappaB signaling pathways. J Cell Biochem 109: 726-736, 2010.

36. Zeng Q, Li S, Chepeha DB, Giordano TJ, Li J, Zhang H, Polverini PJ, Nor J, Kitajewski J and Wang CY: Crosstalk between tumor and endothelial cells promotes tumor angiogenesis by MAPK activation of Notch signaling. Cancer Cell 8: 13-23, 2005.

37. Zavadil J, Cermak L, Soto-Nieves $\mathrm{N}$ and Böttinger EP: Integration of TGF-beta/Smad and Jagged1/Notch signalling in epithelial-to-mesenchymal transition. EMBO J 23: 1155-1165, 2004.

38. Li D, Masiero M, Banham AH and Harris AL: The notch ligand JAGGED1 as a target for anti-tumor therapy. Front Oncol 4: 254, 2014.

39. Fiaschetti G, Schroeder C, Castelletti D, Arcaro A, Westermann F, Baumgartner M, Shalaby T and Grotzer MA: NOTCH ligands JAG1 and JAG2 as critical pro-survival factors in childhood medulloblastoma. Acta Neuropathol Commun 2: 39, 2014. 